\title{
RESISTENCIA ANTIMICROBIANA DE Salmonella spp AISLADA DE ALIMENTOS DE ORIGEN ANIMAL PARA CONSUMO HUMANO
}

\author{
Adriana Quesada1,a, Gabriel A. Reginatto ${ }^{2, b}$, Ayelen Ruiz Español2,b, Lisandro D. Colantonio ${ }^{3,4, b}$, \\ María Soledad Burrone ${ }^{1, c}$
}

\begin{abstract}
RESUMEN
Objetivos. Analizar la información disponible sobre la resistencia antimicrobiana de aislamientos de Salmonella spp de alimentos de origen animal para consumo humano en América Latina. Materiales y métodos. Se realizó una revisión sistemática de estudios epidemiológicos observacionales realizados en América Latina entre los años 2003 y 2014 en las bases de datos PubMed y LILACS. Se excluyeron estudios realizados como parte de estudio de brotes o casos de infecciones en humanos. Tres revisores de forma independiente participaron en la selección de estudios. Además, se realizó la evaluación de calidad a los estudios incluidos. Resultados. Un total de 25 estudios cumplieron con los criterios de inclusión. Los estudios incluidos fueron realizados en Brasil, México, Colombia, Argentina y Venezuela. Los aislamientos de Salmonella spp se obtuvieron principalmente de alimentos de origen avícola, porcino y vacuno, siendo Salmonella typhimurium y Salmonella enteritidis los serotipos que se aislaron con mayor frecuencia (17 y 11 estudios, respectivamente). En 23 de los estudios, Salmonella spp fue resistente a más de un antibiótico, incluyendo ácido nalidíxico, estreptomicina, tetraciclina, cloranfenicol, ampicilina, trimetoprim/sulfametoxazol, gentamicina, ciprofloxacina y cefalosporinas. Conclusiones. Los aislamientos de Salmonella spp obtenidos de alimentos de origen animal para consumo humano en los países analizados presentan con frecuencia resistencia a múltiples antibióticos. Es importante que más países en América Latina realicen y publiquen estudios sobre la resistencia de Salmonella spp para establecer y monitorear estrategias de control adecuadas.
\end{abstract}

Palabras clave: Salmonella; Farmacorresistencia bacteriana; Salud pública; Alimentos de origen animal (fuente: DeCS BIREME

\section{ANTIMICROBIAL RESISTANCE OF Salmonella spp ISOLATED ANIMAL FOOD FOR HUMAN CONSUMPTION}

\begin{abstract}
Objectives. To analyze all information available on antimicrobial-resistant Salmonella species isolated from foods of animal origin that are used for human consumption in Latin America. Materials and methods. A systematic review of observational epidemiological studies conducted in Latin America between 2003 and 2014 was carried out using the PubMed and LILACS databases. Studies conducted as part of analyses of outbreaks or cases of human infection were not included. Three reviewers independently participated in the study selection. Additionally, the studies included underwent quality assessment. Results. A total of 25 studies met the inclusion criteria. The studies included were conducted in Brazil, Mexico, Colombia, Argentina, and Venezuela. Salmonella spp. isolates were obtained mainly from animal-based foods derived from cattle, swine, and poultry, revealing that Salmonella typhimurium and S. enteritidis were the most frequently isolated serotypes (17 and 11 studies, respectively). In 23 studies, Salmonella spp. showed resistance to more than one antibiotic, including nalidixic acid, streptomycin, tetracycline, chloramphenicol, ampicillin, trimethoprim-sulfamethoxazole, gentamicin, ciprofloxacin, and cephalosporins. Conclusions. Salmonella spp. isolates obtained mainly from animal-based foods for human consumption in the countries analyzed often show resistance to several antibiotics. It is important that more countries in Latin America carry out and publish studies on Salmonella spp. resistance in order to establish and monitor adequate control strategies.
\end{abstract}

Key words: Salmonella; Drug resistance, bacterial; Public health; Foods of animal origin (source: MeSH NLM).

\section{INTRODUCCIÓN}

La intoxicación alimentaria ocasionada por bacterias del género Salmonella es una de las zoonosis de mayor prevalencia en países desarrollados y una de las principales causas de enfermedades gastrointestinales en el hombre ${ }^{(1)}$. El número estimado de infecciones humanas por Salmonella spp es superior a 93800000 casos

\footnotetext{
Escuela de Salud Pública, Facultad de Ciencias Médicas, Universidad Nacional de Córdoba. Córdoba, Argentina.

Facultad de Ciencias Médicas, Universidad Nacional de Córdoba. Córdoba, Argentina.

Facultad de Medicina, Universidad de Buenos Aires. Buenos Aires, Argentina.

Department of Epidemiology. School of Public Health. University of Alabama at Birmingham. Alabama, EE. UU.

Médico veterinario, ${ }^{\mathrm{b}}$ médico, ${ }^{\mathrm{c}}$ médico, magíster en Salud Pública.

Recibido: 23-07-15 Aprobado: 09-03-16
}

Citar como: Quesada A, Reginatto GA, Ruiz Español A, Colantonio LD, Burrone MS. Resistencia antimicrobiana de Salmonella spp aislada de alimentos de origen animal para consumo humano. Rev Peru Med Exp Salud Publica. 2016;33(1):32-44. doi:10.17843/rpmesp.2016.331.1899 
anuales, con 155000 muertes al año en todo el mundo (2). En América Latina, Asia y África, la incidencia reportada de salmonelosis es de 200 a 500 casos por 100000 habitantes por año ${ }^{(3)}$. La transmisión de Salmonella spp de persona a persona es poco frecuente, por lo que se considera que los alimentos son la principal fuente de exposición humana ${ }^{(4)}$. Se estima que el $95 \%$ de las infecciones están asociadas con alimentos de origen animal (5).

En los últimos años, diversos estudios han reportado un aumento de la resistencia antimicrobiana en cepas de Salmonella spp aisladas de alimentos de origen animal (6). La creciente resistencia antimicrobiana de Salmonella spp ha sido atribuida al uso extenso de antibióticos, tanto en la terapéutica humana como animal ${ }^{(7)}$. En la industria pecuaria en particular, los antibióticos no solo son utilizados con fines terapéuticos, sino que, además, se utilizan como promotores de crecimiento en dosis subterapéuticas durante largos periodos ${ }^{(6,8)}$.

El presente trabajo tuvo como objetivo revisar los estudios de resistencia antimicrobiana de Salmonella spp aislada de alimentos de origen animal en países de América Latina, con el fin de identificar cuáles son los serotipos de Salmonella spp más frecuentes y los antibióticos a los que presentan resistencia.

\section{MATERIALES Y MÉTODOS}

Se realizó una revisión sistemática de estudios epidemiológicos observacionales sobre la resistencia antimicrobiana de aislamientos de Salmonella spp obtenidos de alimentos de origen animal en América Latina.

\section{CRITERIOS DE INCLUSIÓN Y EXCLUSIÓN}

Se incluyeron estudios descriptivos observacionales realizados en países de América Latina que reportaron la susceptibilidad a antimicrobianos en aislamientos bacterianos correspondientes a las especies del género Salmonella obtenidos de alimentos de origen animal destinados a consumo humano. Para este estudio se utilizó la definición de América Latina propuesta por la División de Población de la Comisión Económica para América Latina y el Caribe (CEPAL), Naciones Unidas, la cual incluye: Argentina, Bolivia, Brasil, Chile, Colombia, Costa Rica, Cuba, República Dominicana, Ecuador, El Salvador, Guatemala, Haití, Honduras, México, Nicaragua, Panamá, Paraguay, Perú, Uruguay y Venezuela ${ }^{(9)}$.

Se incluyeron estudios publicados a partir del año 2003 en idioma inglés, español y portugués. El análisis de estudios publicados a partir del año 2003 fue considerado apropiado para esta revisión, dado que la epidemiología actual de la resistencia antibacteriana por Salmonella spp puede ser diferente a la reportada en estudios más antiguos.
Para la presente revisión sistemática, la definición de alimentos de origen animal destinados a consumo humano incluye: carne de pollo, carne de cerdo, carne de res, embutidos, huevos, leche y sus derivados. Además, se incluyeron estudios en los que se analizaron animales faenados, incluyendo carcasa y animal en canal.

Se excluyeron estudios en los que se analizaron alimentos de origen animal junto a otro tipo de muestras no relevantes para la presente revisión sistemática, y que no reportaron resultados por separado para las muestras obtenidas de alimentos de origen animal. Además, se excluyeron estudios que reportaron resultados de pruebas antimicrobianas de Salmonella spp aislada a partir de estudios de brotes de intoxicación alimentaria en humanos o a partir de casos clínicos.

\section{ESTRATEGIA DE BÚSQUEDA}

La identificación de estudios se realizó a través de una búsqueda bibliográfica en las bases de datos PubMed (Biblioteca Nacional de Medicina, Institutos Nacionales de Salud, Estados Unidos) y Literatura Latinoamericana y del Caribe en Ciencias de la Salud (LILACS, Biblioteca Virtual en Salud, Centro Latinoamericano y del Caribe de Información en Ciencias de la Salud, Organización Panamericana de la Salud). PubMed es una de las bases de datos más importantes sobre publicaciones médicas a nivel mundial, mientras que LILACS es la principal base de datos de publicaciones sobre salud en América Latina.

Para este estudio, dos revisores ( $A Q$ y MSB) crearon y refinaron estrategias de búsqueda para cada base de datos empleando una combinación de términos relacionados con el tema (Anexo 1). Para la búsqueda en la base de datos PubMed se utilizaron términos MeSH (del inglés Medical Subject Headings), y para la base de datos LILACS se utilizaron términos DeCS (Descriptores en Ciencias de la Salud). La búsqueda de los estudios se realizó en mayo del 2014.

\section{SELECCIÓN DE ESTUDIOS}

La selección de estudios se realizó en dos etapas. En la primera etapa, tres revisores en forma independiente (AR, GR y $A Q$ ) analizaron los títulos y resúmenes de todas las referencias recuperadas de PubMed y LILACS utilizando las estrategias de búsqueda. Los revisores seleccionaron publicaciones potencialmente relevantes teniendo en cuenta los criterios de inclusión definidos previamente. En la segunda etapa de la revisión, los tres revisores de forma independiente analizaron el texto completo de todas las publicaciones identificadas como potencialmente relevantes en la etapa previa, utilizando una lista de cotejo desarrollada para tal fin. Cuando hubo desacuerdo, los revisores evaluaron nuevamente el estudio para tratar de alcanzar un consenso. 
En cada etapa, la selección de estudios se realizó por consenso entre los tres revisores. En los casos en que no se alcanzó consenso, un cuarto evaluador (MSB) actuó como árbitro y tomó la decisión final. El proceso de búsqueda y selección de artículos se resumió usando un esquema PRISMA (del inglés Preferred Reporting Items for Systematic Reviews and Meta-Analyses) ${ }^{(10)}$.

\section{EXTRACCIÓN DE DATOS}

La extracción de datos de los estudios seleccionados en esta revisión se realizó utilizando un formulario estructurado que contiene la siguiente información: autores, fecha de publicación, país en el que se realizó el estudio, alimento estudiado (por ejemplo carne de pollo, carne de res, carne de cerdo, embutidos, huevos y sus derivados), serotipo de Salmonella spp analizado, número de aislamientos estudiados, prueba de susceptibilidad, antibióticos analizados y los resultados de las pruebas de susceptibilidad antimicrobiana. Los antibióticos analizados en los estudios incluidos se agruparon de acuerdo al nombre genérico del principio activo y se organizaron por clases. La resistencia antimicrobiana se definió de acuerdo a las pruebas de susceptibilidad antimicrobiana utilizadas, teniendo en cuenta los parámetros empleados en cada estudio.

\section{EVALUACIÓN DE CALIDAD DE LOS ESTUDIOS}

Existen pocas herramientas para la evaluación de la calidad de estudios epidemiológicos observacionales de corte transversal. Para la evaluación de los estudios incluidos, se adaptó un instrumento propuesto por Ciapponi para la lectura crítica y la evaluación de estudios epidemiológicos transversales y de series de casos ${ }^{(11)}$.

El instrumento final utilizado en esta revisión incluye un total de seis puntos y 14 preguntas (Anexo 2). Los puntos incluidos en el instrumento de evaluación de calidad son: definición del objetivo, muestras analizadas, definición y medición de las variables principales, resultados, conclusiones, y conflictos de intereses.

Al finalizar la evaluación de la calidad de los estudios, se realizó la valoración sumaria. Un revisor clasificó cada estudio en las siguientes categorías: de alta calidad, calidad media o calidad baja, teniendo en cuenta la valoración de las preguntas individuales.

\section{ANÁLISIS ESTADÍSTICO}

Como medida del acuerdo inicial entre los revisores (antes de la búsqueda de consenso), se estimó el estadístico kappa de Cohen ${ }^{(12)}$. Este estadístico se utilizó para medir el grado de acuerdo entre distintos evaluadores más allá de lo esperado por simple azar.
Se considera que valores de kappa entre 0 y 0,4 indican un grado de acuerdo mínimo entre los revisores. Valores de kappa de 0,4-0,6; 0,6-0,8 y 0,8-1,0 indican un grado de acuerdo moderado, substancial y casi perfecto, respetivamente ${ }^{(13)}$. Los estadísticos kappa fueron calculados para cada etapa de la revisión por separado y en forma global para todos los revisores.

Se analizaron las características de los estudios incluidos así como la resistencia antimicrobiana de Salmonella spp a cada antibiótico por separado. Además de analizar la resistencia en forma general, se utilizaron tablas cruzadas para analizar la resistencia a cada antibiótico de acuerdo con las características de los estudios, incluyendo país de realización, año de publicación y tipo de alimento analizado. La información de los estudios se sintetizó usando medidas de frecuencia. Para el análisis estadístico se utilizó el programa STATA versión 11.2 (STATA Corp, College Station, Texas).

\section{RESULTADOS}

La Figura 1 muestra el esquema PRISMA que resume el proceso de búsqueda y selección de los artículos. El acuerdo inicial entre los revisores, luego de la revisión de las referencias usando el título y resumen fue substancial (kappa 0,74; IC 95\%: 0,70-0,81). Después de analizar el texto completo y aplicar los criterios de inclusión, se seleccionaron 25 estudios (kappa 0,85; IC 95\%: 0,74-1,00).

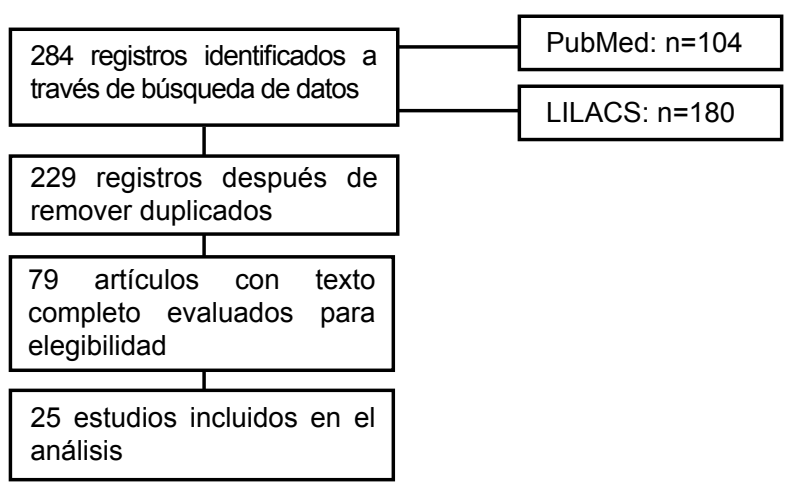

Figura 1. Esquema PRISMA de la búsqueda y selección realizada de los 25 artículos incluidos en la revisión sistemática. LILACS (Literatura Latinoamericana y del Caribe en Ciencias de la Salud), PRISMA (Preferred Reporting Items for Systematic Reviews and Meta-Analyses).

La Tabla 1 muestra las características de los estudios incluidos. Del total de estos, veinte fueron publicados en idioma inglés, tres en español y dos en portugués. Los estudios se realizaron en cinco países de América Latina: Brasil (doce estudios); México (ocho estudios); 
Tabla 1. Características de los 25 estudios incluidos en la revisión sistemática (organizados por país de realización)

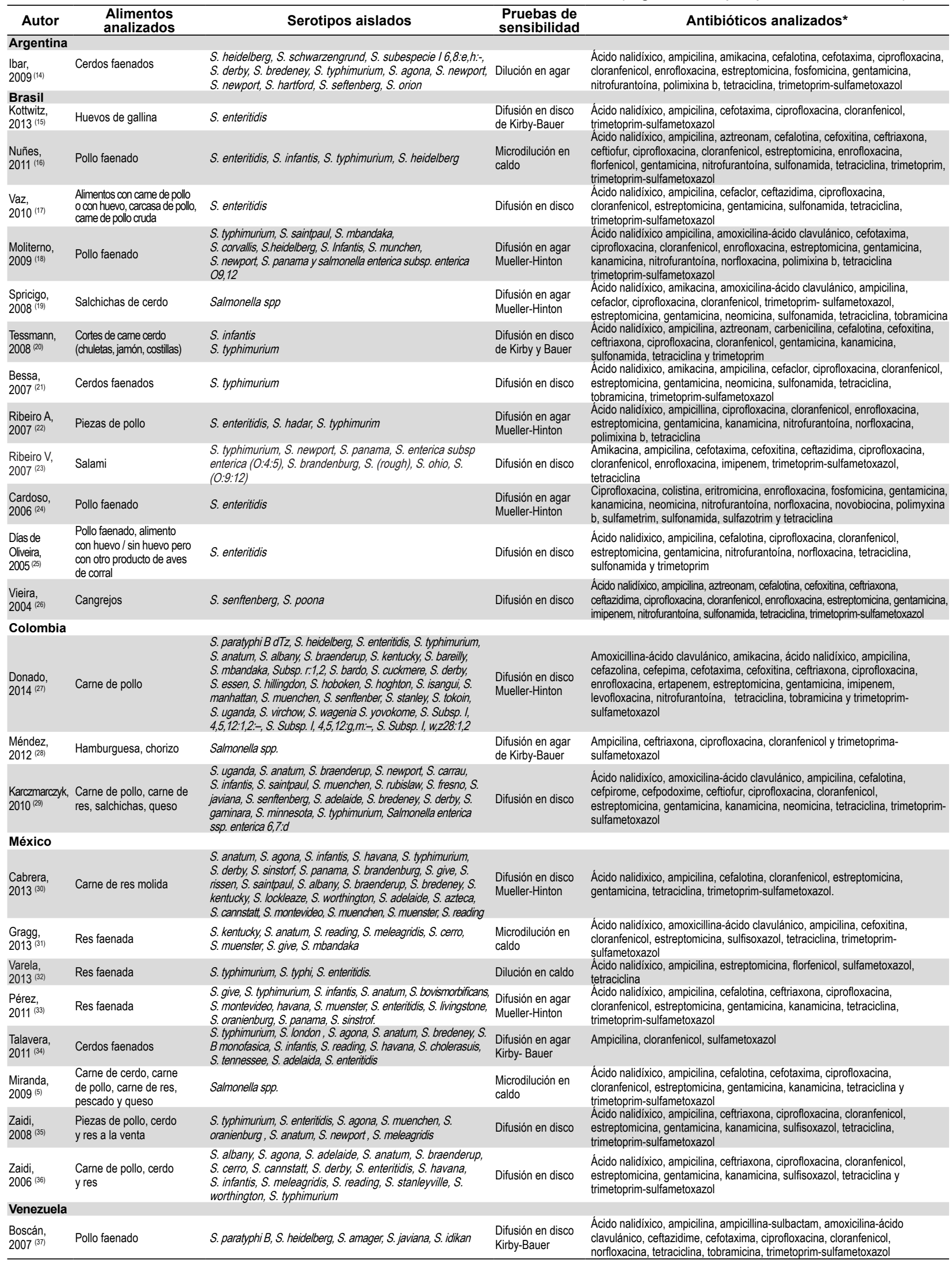

*Se listan solo los antibióticos a los cuales los aislamientos de Salmonella spp fueron resistentes. 
Colombia (tres estudios); Argentina y Venezuela (un estudio cada uno). De acuerdo al año de publicación, un estudio fue publicado en 2004; 3 en 2005-2006; 7 en $2007-2008,5$ en 2009-2010, 4 en 2011-2012 y 5 en 2013-2014.

En 17 estudios (68\%) solo se analizó un tipo de alimento. Con respecto a los alimentos analizados y en los cuales se aisló Salmonella spp, estos fueron: carne de pollo (seis estudios), carne de cerdo (cuatro estudios), carne de res (tres estudios), salchichas, salami, huevos de gallina y cangrejos (un estudio cada uno). Los estudios restantes analizaron más de un tipo de alimento, agrupados de la siguiente manera: carne de pollo, cerdo y res (dos estudios); carne de pollo o huevos; carne de pollo y alimentos con huevo u otro producto de aves de corral; hamburguesa y chorizo; carne de pollo, res, salchicha y queso; ganado faenado; y carne de cerdo, pollo, res, pescado y queso (un estudio cada combinación de alimentos).

Los serotipos que se aislaron con mayor frecuencia fueron S. typhimurium, aislado en 17 estudios, seguido por S. enteritidis, en 11 estudios. En dos estudios no se reportaron el serotipo de Salmonella spp aislado.

El método de difusión en agar fue aplicado como prueba de susceptibilidad antimicrobiana en 20 estudios (80\%), seguido del método de microdilución en caldo (tres estudios), mientras que los métodos de dilución en caldo y dilución en agar se utilizaron en un estudio cada uno.

Los 25 estudios incluidos en la presente revisión analizaron un total de 46 antibióticos diferentes (Tabla 2). Un único estudio (Vieira, 2004) reportó que las cepas de Salmonella spp analizadas fueron sensibles a todos los antibióticos puestos a prueba, aunque presentaron resistencia intermedia a tetraciclina y ácido nalidíxico. Otro estudio (Kottwitz, 2013), reportó resistencia de Salmonella spp a un único antibiótico, ácido nalidíxico. En los restantes 23 estudios, las cepas de Salmonella spp presentaron resistencia a más de un antibiótico. Las cepas de Salmonella spp aisladas mostraron resistencia frecuente a ácido nalidíxico (19 de 21 estudios); ampicilina (15 de 24 estudios); tetraciclina (20 de 22 estudios) y cloranfenicol (15 de 22 estudios). En cuanto a antibióticos de uso clínico para el tratamiento de la salmonelosis, las cepas de Salmonella spp fueron resistentes a ciprofloxacina en 8 de 20 estudios. En cuanto a las cefalosporinas de tercera generación, los aislamientos de Salmonella spp fueron resistentes a cefotaxima en 1 de 7 estudios y a ceftriaxona en 4 de 8 estudios.

Se observó la tendencia a una menor resistencia a algunos antibióticos como gentamicina, ciprofloxacina y ampicilina en estudios realizados en Brasil en comparación con México y otros países (Tabla 3). No se observaron diferencias substanciales en la resistencia a antibióticos cuando los estudios fueron analizados de acuerdo al año de publicación (Tabla 4) o al tipo de alimento investigado (Tabla 5).

\section{RESULTADOS EVALUACIÓN DE CALIDAD}

En total, 19 estudios (76\%) fueron evaluados como de calidad alta o media en los seis puntos analizados (Figura 2). En cuanto a los conflicto de interés, estos no se reportaron en seis estudios y fueron valorados como de baja calidad en este punto.

\section{DISCUSIÓN}

En la presente revisión se analizaron estudios sobre resistencia antimicrobiana de Salmonella spp aisladas en alimentos de origen animal para consumo humano en América Latina. Los estudios identificados se concentraron en un bajo número de países de la región. En la mayoría de los estudios, los aislamientos de Salmonella spp presentaron resistencia a múltiples antibióticos. Estos resultados sugieren que los aislamientos de Salmonella spp obtenidos de alimentos de origen animal para consumo humano en los países estudiados con frecuencia presentan resistencia antimicrobiana.

Los aislamientos de Salmonella spp aislados en los estudios incluidos mostraron, con frecuencia, resistencia a antibióticos que son usados como primera opción en el tratamiento de salmonelosis en humanos, como cloranfenicol, ciprofloxacina, trimetoprim-sulfametoxazol y ceftriaxona. De acuerdo con los resultados, los aislamientos de Salmonella spp presentaron resistencia a cefotaxima con menor frecuencia que a otros antibióticos de uso frecuente en la salmonelosis, por lo que este antibiótico podría resultar la mejor opción terapéutica para iniciar un tratamiento empírico. Sin embargo, estos resultados deben ser confirmados en un mayor número de estudios.

Las cepas de Salmonella spp aisladas con mayor frecuencia fueron $S$. typhimurium y $S$. enteritidis. Estos serotipos han sido reconocidos como los principales agentes etiológicos de infecciones de origen alimentario en seres humanos ${ }^{(38)}$. S. enteritidis sigue siendo el serotipo más frecuentemente aislado en África, Asia y Europa, y el segundo serotipo más común en Norteamérica y Oceanía. En forma global, S. enteritidis representa el $43,5 \%$ del total de aislamientos de Salmonella spp. Los resultados obtenidos sugieren que S. typhimurium y $S$. enteritidis son también responsables de un número significativo de aislamientos de Salmonella spp en alimentos de origen animal en América Latina. 
Tabla 2. Antibióticos analizados y resultados de las pruebas de resistencia en los 25 estudios incluidos en la revisión sistemática

\begin{tabular}{|c|c|c|}
\hline Antibióticos & $\begin{array}{l}\text { Cantidad de estudios en los que los } \\
\text { aislamientos fueron resistentes }\end{array}$ & $\begin{array}{c}\text { Cantidad de estudios en los que se } \\
\text { analizó cada antibiótico }\end{array}$ \\
\hline \multicolumn{3}{|l|}{ Aminoglucósidos } \\
\hline Gentamicina & 8 & 18 \\
\hline Estreptomicina & 15 & 18 \\
\hline Kanamicina & 7 & 9 \\
\hline Amikacina & 1 & 5 \\
\hline Neomicina & 3 & 4 \\
\hline Tobramicina & 2 & 4 \\
\hline \multicolumn{3}{|l|}{ Quinolonas } \\
\hline Ácido nalidíxico & 19 & 21 \\
\hline Ciprofloxacina & 8 & 20 \\
\hline Norfloxacina & 2 & 5 \\
\hline Levofloxacina & 1 & 1 \\
\hline \multicolumn{3}{|l|}{ Fluoroquinolonas } \\
\hline Enrofloxacina & 7 & 8 \\
\hline \multicolumn{3}{|l|}{ Penicilinas } \\
\hline Ampicilina & 15 & 24 \\
\hline Amoxicilina-Ac. clavulánico & 4 & 5 \\
\hline Ampicilina-sulbactam & 0 & 1 \\
\hline \multicolumn{3}{|l|}{ Tetraciclinas } \\
\hline Tetraciclina & 20 & 22 \\
\hline \multicolumn{3}{|l|}{ Fenicoles } \\
\hline Cloranfenicol & 15 & 22 \\
\hline Florfenicol & 2 & 2 \\
\hline \multicolumn{3}{|l|}{ Trimetoprim-Sulfametoxazol } \\
\hline Trimetoprim-Sulfametoxazol & 14 & 19 \\
\hline \multicolumn{3}{|l|}{ Sulfonamidas } \\
\hline Sulfonamida & 7 & 8 \\
\hline Sulfisoxazol & 2 & 4 \\
\hline Sulfametrim & 0 & 1 \\
\hline Sulfazotrim & 0 & 1 \\
\hline Sulfametoxazol & 2 & 2 \\
\hline \multicolumn{3}{|l|}{ Nitrofuranos } \\
\hline Nitrofurantoína & 6 & 8 \\
\hline \multicolumn{3}{|l|}{ Cefalosporinas } \\
\hline Cefalotina (1. a generación) & 5 & 9 \\
\hline Cefazolina (1. ${ }^{a}$ generación) & 1 & 1 \\
\hline Cefaclor (2. a generación) & 1 & 2 \\
\hline Cefotaxima (3. a generación) & 1 & 7 \\
\hline Ceftriaxona (3. a generación) & 4 & 8 \\
\hline Cefpodoxime (3. a generación) & 0 & 1 \\
\hline Ceftazidima (3. ${ }^{a}$ generación) & 1 & 4 \\
\hline Ceftiofur (3. ${ }^{a}$ generación) & 2 & 2 \\
\hline Cefepima (4. a generación) & 1 & 1 \\
\hline Cefpirome & 1 & 1 \\
\hline \multicolumn{3}{|l|}{ Cefaminas } \\
\hline Cefoxitina & 3 & 6 \\
\hline \multicolumn{3}{|l|}{ Betalactámicos } \\
\hline Aztreonam & 0 & 4 \\
\hline \multicolumn{3}{|l|}{ Polipeptídicos } \\
\hline Polimixina b & 0 & 4 \\
\hline Colistina & 1 & 1 \\
\hline \multicolumn{3}{|c|}{ Inhibidores de la dihidrofolato reductasa } \\
\hline Trimetoprim & 2 & 3 \\
\hline \multicolumn{3}{|l|}{ Fosfonatos } \\
\hline Fosfomicina & 1 & 2 \\
\hline Carboxipenicilinas & & \\
\hline Carbenicilina & 1 & 1 \\
\hline Macrólidos & & \\
\hline Eritromicina & 1 & 1 \\
\hline Carbapenems & & \\
\hline Imipenem & 0 & 3 \\
\hline Ertapenem & 0 & 1 \\
\hline Aminocumarinos & & \\
\hline Novobiocina & 1 & 1 \\
\hline
\end{tabular}


Tabla 3. Resultados de la resistencia antimicrobiana de los aislamientos de Salmonella spp de acuerdo al tipo de antibiótico y país de realización del estudio*

\begin{tabular}{|c|c|c|c|c|c|}
\hline Antibiótico & Brasil** $^{* *}$ & México** & Colombia** & Argentina** & Venezuela** \\
\hline \multicolumn{6}{|l|}{ Aminoglucósidos } \\
\hline Gentamicina & $2 / 11$ & $4 / 5$ & $2 / 2$ & --- & --- \\
\hline Estreptomicina & $5 / 8$ & $7 / 7$ & $2 / 2$ & $1 / 1$ & --- \\
\hline Kanamicina & $2 / 4$ & $4 / 4$ & $1 / 1$ & --- & --- \\
\hline \multicolumn{6}{|l|}{ Quinolonas } \\
\hline Ácido nalidíxico & $9 / 10$ & $6 / 7$ & $2 / 2$ & $1 / 1$ & $1 / 1$ \\
\hline Ciprofloxacina & $3 / 12$ & $3 / 4$ & $1 / 3$ & --- & $1 / 1$ \\
\hline \multicolumn{6}{|l|}{ Fluoroquinolonas } \\
\hline Enrofloxacina & $6 / 6$ & --- & $1 / 1$ & $0 / 1$ & --- \\
\hline \multicolumn{6}{|l|}{ Penicilinas } \\
\hline Ampicilina & $5 / 11$ & $7 / 8$ & $2 / 3$ & $1 / 1$ & $0 / 1$ \\
\hline \multicolumn{6}{|l|}{ Tetraciclinas } \\
\hline Tetraciclina & 9/11 & $7 / 7$ & $2 / 2$ & $1 / 1$ & $1 / 1$ \\
\hline \multicolumn{6}{|l|}{ Fenicoles } \\
\hline Cloranfenicol & $5 / 11$ & $6 / 7$ & $2 / 2$ & $1 / 1$ & $1 / 1$ \\
\hline \multicolumn{6}{|l|}{ Trimetoprim-Sulfametoxazol } \\
\hline Trimetoprim-Sulfametoxazol & $5 / 8$ & $5 / 6$ & $2 / 3$ & $1 / 1$ & $1 / 1$ \\
\hline \multicolumn{6}{|l|}{ Sulfonamidas } \\
\hline Sulfonamida & $7 / 8$ & --- & --- & --- & --- \\
\hline \multicolumn{6}{|l|}{ Nitrofuranos } \\
\hline Nitrofurantoína & $4 / 6$ & --- & $1 / 1$ & $1 / 1$ & --- \\
\hline \multicolumn{6}{|l|}{ Cefalosporinas } \\
\hline Cefalotina (1. ${ }^{\mathrm{a}}$ generación) & $2 / 4$ & $2 / 3$ & $1 / 1$ & $0 / 1$ & --- \\
\hline Ceftriaxona (3.a generación) & $1 / 3$ & $2 / 3$ & $1 / 2$ & --- & --- \\
\hline Cefotaxima ( $3 .^{a}$ generación) & $0 / 3$ & $0 / 1$ & $1 / 1$ & $0 / 1$ & $0 / 1$ \\
\hline \multicolumn{6}{|l|}{ Cefaminas } \\
\hline Cefoxitina & $2 / 4$ & $1 / 1$ & $0 / 1$ & --- & --- \\
\hline
\end{tabular}

Tabla 4. Resultados de la resistencia antimicrobiana de los aislamientos de Salmonella spp de acuerdo al tipo de antibiótico y país de realización del estudio*

\begin{tabular}{|c|c|c|c|c|c|c|}
\hline Antibiótico & $2003-2004^{\star \star}$ & $2005-2006^{* *}$ & $2007-2008^{\star \star}$ & $2009-2010^{\star *}$ & $2011-2012^{\star *}$ & $2013-2014^{\star \star}$ \\
\hline \multicolumn{7}{|l|}{ Aminoglucósidos } \\
\hline Gentamicina & $0 / 1$ & $2 / 2$ & $2 / 6$ & $2 / 5$ & $2 / 2$ & $0 / 2$ \\
\hline Estreptomicina & $0 / 1$ & $2 / 2$ & $3 / 4$ & $4 / 5$ & $2 / 2$ & $4 / 4$ \\
\hline Kanamicina & --- & $2 / 2$ & $1 / 3$ & $3 / 3$ & $1 / 1$ & --- \\
\hline \multicolumn{7}{|l|}{ Quinolonas } \\
\hline Ácido nalidíxico & $0 / 1$ & $2 / 2$ & $5 / 6$ & $5 / 5$ & $2 / 2$ & $5 / 5$ \\
\hline Ciprofloxacina & $0 / 1$ & $0 / 2$ & $3 / 7$ & $2 / 5$ & $1 / 3$ & $2 / 2$ \\
\hline \multicolumn{7}{|l|}{ Fluoroquinolonas } \\
\hline Enrofloxacina & --- & $1 / 1$ & $2 / 2$ & $1 / 2$ & $2 / 2$ & $1 / 1$ \\
\hline \multicolumn{7}{|l|}{ Penicilinas } \\
\hline Ampicilina & $0 / 1$ & $1 / 2$ & $5 / 7$ & $4 / 5$ & $3 / 4$ & $2 / 5$ \\
\hline \multicolumn{7}{|l|}{ Tetraciclinas } \\
\hline Tetraciclina & $0 / 1$ & $3 / 3$ & $7 / 7$ & $4 / 5$ & $2 / 2$ & $4 / 4$ \\
\hline \multicolumn{7}{|l|}{ Fenicoles } \\
\hline Cloranfenicol & $0 / 1$ & $1 / 2$ & $5 / 7$ & $3 / 6$ & $3 / 3$ & $2 / 3$ \\
\hline \multicolumn{7}{|l|}{ Trimetoprim/Sulfametoxazol } \\
\hline Trimetoprim/Sulfametoxazol & $0 / 1$ & $1 / 1$ & $4 / 5$ & $3 / 5$ & $3 / 3$ & $3 / 4$ \\
\hline \multicolumn{7}{|l|}{ Sulfonamidas } \\
\hline Sulfonamida & $0 / 1$ & $2 / 2$ & $3 / 3$ & $1 / 1$ & $1 / 1$ & --- \\
\hline \multicolumn{7}{|l|}{ Nitrofuranos } \\
\hline Nitrofurantoína & $0 / 1$ & $2 / 2$ & $1 / 1$ & $2 / 2$ & $1 / 1$ & $0 / 1$ \\
\hline \multicolumn{7}{|l|}{ Cefalosporinas } \\
\hline Cefalotina ( $1^{\text {ra }}$ generación) & $0 / 1$ & $0 / 1$ & $1 / 1$ & $2 / 3$ & $2 / 2$ & $0 / 1$ \\
\hline Ceftriaxona (3. ${ }^{\text {a generación) }}$ & $0 / 1$ & $1 / 1$ & $1 / 2$ & -- & $1 / 3$ & $1 / 1$ \\
\hline Cefotaxima (3. ${ }^{a}$ generación) & --- & --- & $0 / 2$ & $0 / 3$ & --- & $1 / 2$ \\
\hline \multicolumn{7}{|l|}{ Cefaminas } \\
\hline Cefoxitina & $0 / 1$ & --- & $0 / 2$ & --- & $1 / 1$ & $2 / 2$ \\
\hline
\end{tabular}

* En la tabla solo se listan los antibióticos analizados en más de seis estudios.

** Cantidad de estudios en los que los aislamientos fueron resistentes / cantidad de estudios en los que se analizó cada antibiótico 
Tabla 5. Resultados de la resistencia antimicrobiana de los aislamientos de Salmonella spp de acuerdo al tipo de antibiótico y alimento analizado*

\begin{tabular}{|c|c|c|c|c|c|c|}
\hline Antibiótico & $\begin{array}{c}\text { Carne de } \\
\text { pollo**t }\end{array}$ & $\begin{array}{c}\text { Carne de } \\
\text { res }^{* * \ddagger}\end{array}$ & $\begin{array}{l}\text { Carne de } \\
\text { cerdo**§ }\end{array}$ & $\begin{array}{c}\text { Salchicha de } \\
\text { cerdo** }^{* *}\end{array}$ & Salami $^{* *}$ & Huevos** \\
\hline \multicolumn{7}{|l|}{ Aminoglucósidos } \\
\hline Gentamicina & $1 / 7$ & $1 / 2$ & $1 / 5$ & -- & --- & --- \\
\hline Estreptomicina & $5 / 4$ & $4 / 4$ & $2 / 2$ & --- & --- & --- \\
\hline Kanamicina & $2 / 3$ & $1 / 1$ & -- & --- & --- & --- \\
\hline \multicolumn{7}{|l|}{ Quinolonas } \\
\hline Ácido nalidíxico & $5 / 6$ & $4 / 4$ & $2 / 3$ & $1 / 1$ & --- & $1 / 1$ \\
\hline Ciprofloxacina & $2 / 8$ & $1 / 1$ & $1 / 3$ & $1 / 1$ & --- & --- \\
\hline \multicolumn{7}{|l|}{ Fluoroquinolonas } \\
\hline Enrofloxacina & $5 / 4$ & --- & --- & --- & $1 / 1$ & --- \\
\hline \multicolumn{7}{|l|}{ Penicilinas } \\
\hline Ampicilina & $2 / 6$ & $3 / 3$ & $3 / 3$ & $1 / 1$ & $1 / 1$ & --- \\
\hline \multicolumn{7}{|l|}{ Tetraciclinas } \\
\hline Tetraciclina & $7 / 8$ & $4 / 4$ & $3 / 4$ & $1 / 1$ & $1 / 1$ & --- \\
\hline \multicolumn{7}{|l|}{ Fenicoles } \\
\hline Cloranfenicol & $2 / 6$ & $1 / 3$ & $3 / 3$ & $1 / 1$ & $1 / 1$ & --- \\
\hline \multicolumn{7}{|l|}{ Trimetoprim-Sulfametoxazol } \\
\hline Trimetoprim-Sulfametoxazol & $4 / 5$ & $3 / 3$ & $1 / 2$ & $1 / 1$ & $1 / 1$ & --- \\
\hline \multicolumn{7}{|l|}{ Sulfonamidas } \\
\hline Sulfonamida & $2 / 3$ & --- & $2 / 2$ & $1 / 1$ & --- & --- \\
\hline \multicolumn{7}{|l|}{ Nitrofuranos } \\
\hline Nitrofurantoína & $5 / 6$ & --- & $1 / 1$ & --- & --- & --- \\
\hline \multicolumn{7}{|l|}{ Cefalosporinas } \\
\hline Cefalotina (1. a generación) & $1 / 2$ & $1 / 2$ & $1 / 2$ & --- & --- & --- \\
\hline Ceftriaxona ( $3 .^{a}$ generación) & $1 / 2$ & --- & --- & --- & --- & --- \\
\hline Cefotaxima ( $3 .^{a}$ generación) & $1 / 3$ & --- & --- & --- & --- & --- \\
\hline \multicolumn{7}{|l|}{ Cefaminas } \\
\hline Cefoxitina & $1 / 2$ & $1 / 1$ & --- & --- & --- & --- \\
\hline
\end{tabular}

* En la tabla solo se listan los antibióticos analizados en más de seis estudios. El análisis se limita a estudios que analizaron un único tipo de alimento.

** Cantidad de estudios en los que los aislamientos fueron resistentes / Cantidad de estudios en los que se analizó cada antibiótico

$\dagger$ Incluye pollo faenado, piezas de pollo a la venta y carne de pollo.

¥ Incluye carne y carne de res molida, y res faenada.

$\S$ Incluye cerdo faenado y carne de cerdo.

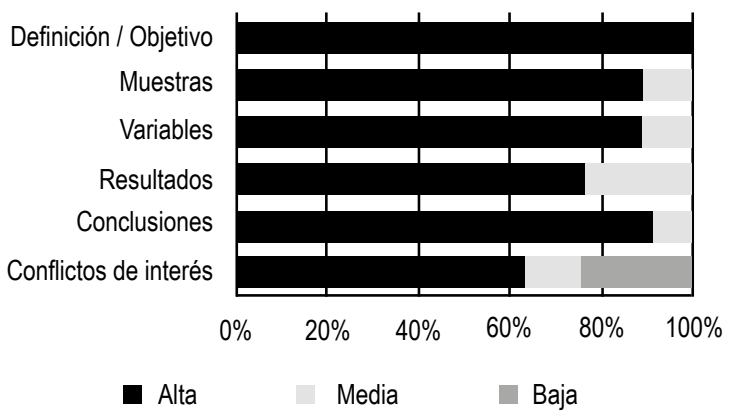

Figura 2. Resultados de la evaluación de calidad de los 25 estudios incluidos en la revisión sistemática de acuerdo a los seis ítems evaluados.
Los resultados obtenidos coinciden con otros estudios previos ${ }^{(7,8,39)}$. Cota realizó una revisión sistemática de estudios de resistencia antimicrobiana de aislamientos de Salmonella spp obtenidos de alimentos de origen animal a nivel mundial (8). En dicho estudio los antibióticos a los que los aislamientos de Salmonella spp presentaron resistencia con mayor frecuencia fueron tetraciclina, estreptomicina, ácido nalidíxico y ampicilina. Otros estudios también han reportado la resistencia de Salmonella spp a antibióticos comúnmente utilizados para el tratamiento de la salmonelosis en humanos incluyendo la ciprofloxacina ${ }^{(39)}$.

La resistencia de Salmonella spp y otras bacterias a los antimicrobianos constituye un motivo de preocupación 
en el ámbito de la salud pública a nivel mundial ${ }^{(40)}$. La resistencia a los antimicrobianos puede asociarse al fracaso terapéutico en humanos y alargar la estancia hospitalaria, produciendo aumento en el gasto de los sistemas de salud (40,41). Así mismo, los costos económicos, sociales y emocionales para los pacientes se ven incrementados ${ }^{(41)}$. La elevada frecuencia de resistencia a antibióticos observada en la presente revisión, incluyendo aquellos de uso clínico para el tratamiento de la salmonelosis, indican la necesidad de establecer sistemas de control y vigilancia adecuados para reducir la resistencia en aislamientos de alimentos de origen animal. Se ha sugerido que el uso extenso de antibióticos en humanos, y en particular el uso prolongado de antibióticos en la producción animal, pueden contribuir a la aparición de resistencia a antibióticos ${ }^{(7,8)}$. Por ejemplo, se ha sugerido que la alta frecuencia de resistencia a quinolonas en los aislamientos de Salmonella spp, particularmente en aquellos de origen aviar, se debe al uso frecuente de enrofloxacina y ácido nalidíxico con fines terapéuticos o metafilácticos (i.e., el uso de antibióticos al detectarse los primeros signos de la enfermedad) ${ }^{(6,42)}$.

En América Latina, algunos países han comenzado a implementar alternativas para controlar esta problemática. Por ejemplo, países como Colombia, Argentina y Perú han limitado la comercialización de antibióticos como el cloranfenicol, olaquindox, nitroimidazoles y nitrofuranos para su uso en animales destinados a consumo humano ${ }^{(43)}$. A nivel mundial, otros países ya han tomado medidas para contener la resistencia a antibióticos en aislamientos de alimentos de origen animal. La Agencia de Alimentos y Drogas de Estados Unidos o FDA (Food and Drug Administration) impulsa distintas estrategias para reducir el uso de antibióticos en los animales de consumo, incluyendo promover cambios voluntarios por parte de los productores. Por ejemplo, la FDA promueve el rol del veterinario licenciado para la supervisión del tratamiento, control y prevención de enfermedades en los animales productores de alimentos. La FDA considera que el cambio voluntario de los productores es la forma más eficaz para poner en práctica cambios duraderos orientados a proteger la salud pública y animal ${ }^{(44)}$.

Debido a que la resistencia antimicrobiana constituye un problema a nivel mundial, se ha planteado la necesidad de establecer con urgencia acciones globales lideradas por organismos internacionales como la Organización Mundial de la Salud (OMS). Estas acciones deberían orientarse a reducir el uso de antimicrobianos en todos los sectores de manera integral, haciendo énfasis en la higiene y la prevención de las infecciones, el control de su suministro en la cría de animales (ya sea para estimular el crecimiento o para la prevención de enfermedades), así como promoviendo un uso más racional en la terapéutica en humanos. La OMS podría considerar buscar un tratado legalmente vinculante con el fin de fomentar la cooperación de todos los estados miembros ${ }^{(30)}$.

Las principales fortalezas de la presente revisión incluyen la búsqueda bibliográfica en dos de las principales y más completas bases de datos sobre estudios realizados en América Latina, la utilización de estrategias de búsqueda que fueron refinadas de acuerdo a los criterios de selección definidos a priori, así como la selección de estudios por más de un revisor, minimizando la posibilidad de sesgos y errores durante el proceso de selección. Cabe mencionar que la presente revisión puede no haber identificado todos los estudios realizados en América Latina. Por otra parte, la alta frecuencia de resistencia a antibióticos en la presente revisión podría estar relacionada con un sesgo de publicación, ya que aquellos estudios en los que se demuestra resistencia a antimicrobianos pueden ser publicados con mayor frecuencia que aquellos que no encuentran resistencia. La mayoría de los estudios incluidos no reportó la proporción de aislamientos de Salmonella spp que fueron resistentes sobre el total de aislamientos analizados. Sin embargo, todos ellos reportaron si se encontró resistencia o no en los aislamientos en forma combinada. Por tal motivo, no fue posible realizar un metaanálisis de los resultados de los estudios individuales.

En conclusión, a pesar de la extensa búsqueda bibliográfica realizada, solo se identificaron pocos países de América Latina en dónde se hayan realizado y reportado estudios de resistencia de aislamientos Salmonella spp obtenidos de alimentos de origen animal destinados a consumo humano. Los resultados sugieren que los aislamientos de Salmonella spp obtenidos de alimentos de origen animal para consumo humano en los países estudiados, con frecuencia, presentan resistencia a múltiples antibióticos, incluyendo aquellos de uso clínico. Estos resultados indican la necesidad de estimular la realización y difusión de este tipo de estudios en más países de América Latina, los cuales podrían servir para establecer y monitorear estrategias y programas locales orientados a disminuir la resistencia bacteriana en aislamientos obtenidos de alimentos.

Conflictos de interés: los autores declaran no tener conflictos de interés en la publicación de este artículo.

Fuentes de financiamiento: autofinanciando.

Contribuciones de autoría: $A Q$ participó en la concepción y diseño del artículo, la recolección de datos, revisión de artículos incluidos, análisis e interpretación de los resultados, y redacción del artículo. GAR y ARE han participado en la recolección de datos, revisión y selección de artículos incluidos. MSB y LDC participaron en el diseño del artículo, recolección de datos, asesoría en el análisis e interpretación de los resultados. Además MSB en caso de que no se alcanzara consenso en la selección de los artículos incluidos, actuó como árbitro y tomó la decisión final. Todos los autores realizaron una revisión crítica del artículo y aprobaron su versión final enviada a publicación. 


\section{REFERENCIAS BIBLIOGRÁFICAS}

1. Sánchez M. Determinación de la prevalencia de enterobacterias del género Salmonella spp. en huevos frescos de gallina de empresas avícolas de la provincia de Tungurahua [tesis para bachiller]. Ecuador: Facultad de Medicina Veterinaria y Zootecnia. Universidad Central del Ecuador; 2013.

2. Campioni F, Moratto Bergammini AM, Falcão JP. Genetic diversity, virulence genes and antimicrobial resistance of Salmonella enteritidis isolated from food and humans over a 24-year period in Brazil. Food Microbiol. 2012;32(2):254-64. doi: 10.1016/j.fm.2012.06.008.

3. Cardona-Castro NM, SánchezJiménez MM, Usuga-Silva LY, Arboleda-Naranjo M, Garzón E, Vélez A, et al. Caracterización de dos brotes de fiebre tifoidea en Apartadó, Antioquia. Biomedica. 2007;27(2):236-43. doi: 10.7705/biomedica.v27i2.219

4. Puig Y, Espino M, Leyva V, Martino T, Mendez D, Soto P, et al. Susceptibilidad antimicrobiana en cepas de Salmonella spp. de origen clínico y alimentario. Rev Panam Infectol. 2007;9(3):12-6.

5. Miranda JM, Mondragón AC, Martinez B, Guarddon M, Rodriguez JA. Prevalence and antimicrobial resistance patterns of Salmonella from different raw foods in Mexico. J Food Prot. 2009;72(5):966-71.

6. Rivera LG, Motta PA, Cerón MF, Chimonja FA. Resistencia de la Salmonella a los antimicrobianos convencionales para su tratamiento. Rev CES Med Vet Zootec. 2012; 7 (1): 116-29.

7. Junod T, López-Martin J, Gädicke P. Estudio de susceptibilidad antimicrobiana de Salmonella enterica en muestras de origen animal y alimentario. Rev Med Chile. 2013;141(3):298-304. doi: 10.4067/ S0034-98872013000300003.

8. Cota-Rubio E, Hurtado-Ayala L, Pérez-Morales E, Alcántara-Jurado L. Resistencia a antibióticos de cepas bacterianas aisladas de animales destinados al consumo humano. ReIbCi. 2014;1(1):75-85.

9. Population Division. Economic Commission for Latin America and the Caribbean (ECLAC) [Internet]. Washington, D.C.; ECLAC; [citado el 10 de junio de 2014]. Disponible en: http://www.cepal.org/celade/default. asp?idioma $=$ IN

10. Moher D, Liberati A, TetzlaffJ, Altman DG; PRISMA Group. Preferred reporting items for systematic reviews and meta-analyses: the PRISMA statement. J Clin Epidemiol. 2009;62(10):1006-12. doi: 10.1016/j. jclinepi.2009.06.005.

11. Ciapponi A. Guía de lectura crítica de estudios observacionales en epidemiología. Evid actual práct ambul. 2010;13(4):135-40.

12. Cohen J. A coefficient of agreement for nominal scales. Educ Psychol Meas. 1960;20(1):37-46. doi: $10.1177 / 001316446002000104$

13. Landis JR, Koch GG. The measurement of observer agreement for categorical data. Biometrics. 1977;33(1):159-74.

14. Ibar MP, Vigo G, Piñeyro P, Caffer MI, Quiroga P, Perfumo C, et al. Serovariedades de Salmonella entérica subespecie enterica en porcinos de faena y su resistencia a los antimicrobianos. Rev Argent Microbiol. 2009;41(3):156-62.

15. Kottwitz LB, Leão JA, Back A, Rodrigues Ddos P, Magnani M, Oliveira TC. Commercially laid eggs vs. discarded hatching eggs: contamination by Salmonella spp. Brazil J Microbiol. 2013;44(2):367-70. doi: 10.1590/ S1517-83822013005000036

16. Medeiros MA, Oliveira DC, Rodrigues Ddos P, Freitas DR. Prevalence and antimicrobial resistance of Salmonella in chicken carcasses at retail in 15 Brazilian cities. Rev Panam Salud Publica. 2011;30(6):555-60. doi: 10.1590/ S1020-49892011001200010.

17. Vaz CS, Streck AF, Michael GB, Marks FS, Rodrigues DP, Dos Reis EM, et al. Antimicrobial resistance and subtyping of Salmonella enterica subspecies enterica serovar enteritidis isolated from human outbreaks and poultry in southern Brazil. Poult Sci. 2010; 89(7):1530-6. doi: 10.3382/ps.200900453.

18. Duarte DA, Ribeiro AR, Vasconcelos AM, Santos SB, Silva JV, de Arruda Falcão LS. Occurrence of Salmonella spp. in broiler chicken carcasses and their susceptibility to antimicrobial agents. Braz J Microbiol. 2009;40(3):569-73. doi: 10.1590/ S1517-838220090003000020

19. Spricigo DA, Matsumoto SR, Espíndola ML, Vaz EK, Ferraz SM. Prevalência e perfil de resistência a antimicrobianos de sorovares de Salmonella isolados de lingüiças suínas tipo frescal em Lages, SC. Arq Bras Med Vet Zootec. 2008;60(2):517-20. doi: 10.1590/ S0102-09352008000200040

20. Tessmann C, Zocche F, Lima A, Bassani M, Lopes G, Silva W. Ocorrência e perfil de sensibilidade a antibióticos de Salmonella spp. isolada em cortes de carne suína comercializados em feiras-livres de Pelotas (RS). Bol Cent Pesqui Process Aliment. 2008; 26(2):307-313. doi: 10.5380/cep.v26i2.13294

21. Bessa MC, Michael GB, Canu N, Canal CW, Cardoso M, Rabsch $\mathrm{W}$, et al. Phenotypic and genetic characterization of Salmonella enterica subsp. enterica serovar Typhimurium isolated from pigs in Rio Grande do Sul, Brazil. Res Vet Sci. 2007;83(3):302-10. doi:10.1016/j. rvsc.2007.01.006.

22. Ribeiro AR, Kellermann A, Santos LR, Nascimento VP. Resistência antimicrobiana em Salmonella enteritidis isoladas de amostras clínicas e ambientais de frangos de corte e matrizes pesadas. Arq Bras Med Vet Zootec. 2008; 60(5):1259-62.

23. Ribeiro VB, Andrigheto C, Bersot LS, Barcellos V, Reis EF, Destro MT. Serological and genetic diversity amongst Salmonella strains isolated in a salami processing line. Braz J Microbiol. 2007;38(1):17882. doi: $10.1590 /$ S151783822007000100036.

24. Cardoso M, Ribeiro A, Santos L, Pilotto F, Moraes H, Salle C, et al. Antibiotic resistance in Salmonella enteritidis isolated from broiler carcasses. Braz J Microbiol. 2006;37(3):368-71. doi: 10.1590/ S1517-83822006000300030.

25. Dias de Oliveira S, Siqueira Flores F, dos Santos LR, Brandelli A. Antimicrobial resistance in Salmonella enteritidis strains isolated from broiler carcasses, food, human and poultryrelated samples. Int J Food Microbiol. 
2005; 97(3): 297-305. doi:10.1016/j. ijfoodmicro.2004.04.022.

26. Vieira RH, Lima EA, Sousa DB, Reis EF, Costa RG, Rodrigues Ddos P. Vibrio spp. and Salmonella spp., presence and susceptibility in crabs Ucides cordatus. Rev Inst Med Trop Sao Paulo. 2004;46(4):179-82. doi: 10.1590/ S0036-46652004000400001.

27. Donado-Godoy P, Clavijo V, León M, Arévalo A, Castellanos R, Bernal J, et al. Counts, serovars, and antimicrobial resistance phenotypes of Salmonella on raw chicken meat at retail in Colombia. J Food Prot. 2014;77(2):227-235. doi: 10.4315/0362-028X.JFP-13-276.

28. Méndez IA, Badillo CA, Ortiz G, Faccini AA. Caracterización microbiológica de Salmonella en alimentos de venta callejera en un sector universitario de Bogotá, Colombia. Julio a octubre de 2010. Méd UIS. 2011;24(1):23-9.

29. Karczmarczyk M, Martins M, McCusker M, Mattar S, Amaral L, Leonard N, et al. Characterization of antimicrobial resistance in Salmonella enterica food and animal isolates from Colombia: identification of a qnrB19mediated quinolone resistance marker in two novel serovars. FEMS Microbiol Lett. 2010;313(1):10-9. doi: 10.1111/j.1574-6968.2010.02119.x.

30. Cabrera-Diaz E, Barbosa-Cardenas CM, Perez-Montaño JA, GonzalezAguilar D, Pacheco-Gallardo C, Barba J. Occurrence, serotype diversity, and antimicrobial resistance of Salmonella in ground beef at retail stores in Jalisco state, Mexico. J Food Prot. 2013;76(12):2004-10. doi: 10.4315/0362-028X.JFP-13-109.

31. Gragg SE, Loneragan GH, Nightingale KK, Brichta-Harhay DM, Ruiz $\mathrm{H}$, Elder JR, et al. Substantial withinanimal diversity of Salmonella isolates from lymph nodes, feces, and hides of cattle at slaughter. Appl Environ Microbiol. 2013;79(15):4744-50. doi: 10.1128/AEM.01020-13.
32. Varela-Guerrero JA, Talavera-Rojas M, Gutierrez-Castillo Adel C, ReyesRodriguez NE, Vázquez-Guadarama J. Phenotypic-genotypic resistance in Salmonella spp. isolated from cattle carcasses from the north central zone of the State of Mexico. Trop Anim Health Prod. 2013; 45(4):995-1000. doi: 10.1007/s11250-012-0323-x.

33. Pérez-Montaño JA, Gonzalez-Aguilar D, Barba J, Pacheco-Gallardo C, Campos-Bravo CA, García S, et al. Frequency and antimicrobial resistance of Salmonella serotypes on beef carcasses at small abattoirs in Jalisco State, Mexico. J Food Prot. 2012;75(5):867873. doi: 10.4315/0362-028X.JFP-11423.

34. Talavera M, Varela J, Reyes N, Lagunas $\mathrm{S}$, Valladares $\mathrm{B}$, Alonso $\mathrm{M}$, et al. Resistencia antibiótica de genotipos de cepas de Salmonella spp de cerdos sacrificados en rastros del Estado de México. Vet Mex. 2011; 42(4):269276.

35. Zaidi MB, Clava JJ, Estrada-Garcia MT, Leon V, Vazquez G, Figueroa G, et al. Integrated Food Chain Surveillance System for Salmonella spp. in Mexico. Emerg Infect Dis. 2008;14(3):429-35. doi: 10.3201/eid1403.071057.

36. Zaidi MB, McDermott PF, DedorkaCray P, Leon V, Canche C, Hubert SK, et al. Nontyphoidal Salmonella from human clinical cases, asymptomatic children, and raw retail meats in Yucatan, Mexico. Clin Infect Dis. 2006; 42 (1):21-8. doi: 10.1086/498508

37. Boscán-Duque LA, Arzálluz-Fisher AM, Ugarte C, Sánchez D, Wittum TE, Hoet AE. Reduced susceptibility to quinolones among Salmonella serotypes isolated from poultry at slaughter in Venezuela. J Food Prot. 2007; 70(9):2030-5.

38. De Oliveira FA, Brandelli A, Tondo EC. Antimicrobial resistance in Salmonella enteritidis from foods involved in human salmonellosis outbreaks in southern Brazil. New Microbiologica. 2006;29;49-54.
39. Jurado Jiménez R, Arenas Muñoz C, Doblas Delgado A, Rivero A, Torre Cisneros J. Fiebre tifoidea y otras infecciones por salmonellas. Medicine. 2010;10(52):3497-501. doi 10.1016/S0304-5412(10)70069-X.

40. Red internacional de autoridades de inocuidaddelosalimentos(INFOSAN). Resistencia antimicrobiana a Salmonella [Internet]. Ginebra: OMS; 2005 [citado el 20 de noviembre de 2014] Disponible en: http://www.who.int/foodsafety/ fs_management/No_03_Salmonella Apr05_sp.pdf.

41. Organización Panamericana de la Salud (OPS), Organización Mundial de la Salud (OMS). Mesa redonda sobre la resistencia a los antimicrobianos. contener la resistencia a los antimicrobianos [Internet]. Washington: OPS/OMS; 2011 [citado el 30 de marzo de 2015]. Disponible en: http://wwwpaho.org/HQ/ index.php?option $=$ com_docman\&task $=\mathrm{doc}$ download\&Itemid $=270$ \& gid $=16011$ \&lang=es

42. Sumano H, Gutiérrez L. Problemática del uso de enrofloxacina en la avicultura. Vet Mex [Internet]. 2000 [citado el 29 de enero del 2015];31(2):1-6. Disponible en: http://www.ejournal.unam.mx/rvm/ vol31-02/RVM31209.pdf

43. Carranza C, Vargas J. Uso de medicamentos en avicultura. Avicultores [Internet]. 2012 [citado el 10 de enero de 2015];197:42-45. Disponible en: http://www.fenavi.org/images/stories/ revistaavicultores/libros/revista-197/

44. Food and Drug Administration (FDA) [Internet]. Maryland: FDA; [actualizado el 6 de noviembre de 2105, citado el 5 de junio de 2015]. Disponible en: http://www.fda.gov/AnimalVeterinary/ GuidanceComplianceEnforcement/ GuidanceforIndustry/ucm216939.htm

Correspondencia: Adriana Julieth
Quesada Parra
Dirección: Transversal 64 \# 1-55 Interior
6apartamento 302 (Bogotá, Colombia)
Teléfono: (511) 322-2502718
Correo electrónico:naniquesada@hotmail.com




\section{ANEXOS}

Anexo 1. Estrategia de búsqueda para las bases de datos utilizadas en la revisión sistemática

\section{Estrategia de búsqueda}

"Salmonella"[MeSH Terms] OR "Salmonella"[tiab] OR "Salmonella infections"[MeSH Terms] OR "salmonellosis"[tiab] OR "Salmonella food poisoning"[MeSH Terms] OR "Salmonella typhimurium"[MeSH Terms] OR "Salmonella enteritidis"[MeSH Terms] OR "Salmonella enterica"[MeSH Terms] OR "S. enteritidis"[tiab] OR "S. typhimurium"[tiab] AND "microbial sensitivity tests"[MeSH Terms] OR "Drug Resistance, Bacterial"[MeSH Terms] OR "drug resistance, microbial"[MeSH Terms] OR ((“anti-infective agents"[Pharmacological Action] OR "anti-infective agents"[MeSH Terms] OR ("anti-infective"[tiab] AND "agents"[tiab]) OR "antimicrobial"[tiab] OR "antibiotic"[tiab]) AND ("resistance"[tiab] OR "drug resistance, multiple"[MeSH Terms] OR ("sensitivity"[tiab] AND "tests"[tiab]))) AND "animals"[tiab] OR "animal"[tiab] OR "abattoirs"[MeSH Terms] OR "abattoirs"[tiab] OR "abattoir"[tiab] OR "slaughterhouse"[tiab] OR "slaughterhouses"[tiab] OR "slaughter"[tiab] OR "sacrifice"[tiab] OR ("butcher"[tiab] AND "shop"[tiab]) OR "Food Microbiology"[MeSH terms] OR "Pork"[tiab] OR "sausage"[tiab] OR "poultry"[tiab] OR "pig”[tiab] OR "chicken"[tiab] OR "sausages"[tiab] OR "poultries"[tiab] OR "pigs"[tiab] OR "chickens"[tiab] OR "hens"[tiab] OR "hen"[tiab] OR "eggs"[tiab] OR "egg"[tiab] OR "food pathogen"]tiab] OR "swine"[tiab] OR "food microbiology"[tiab] OR "food surveillance"[tiab] OR "meat"[tiab] OR “Swine Diseases"[MeSH terms] OR "Animal Husbandry"[MeSH terms] OR "Animal Husbandry"[tiab] OR "Poultry Diseases"[MeSH terms] OR "Poultry Diseases"[tiab] OR "Chickens/microbiology"[Mesh] OR "Eggs/ microbiology"[Mesh terms] OR "Meat/microbiology"[Mesh terms] OR "Sheep, Domestic/microbiology"[Mesh terms] OR "Cattle/microbiology"[Mesh terms] OR "Swine/microbiology"[Mesh terms] OR "Poultry/microbiology"[Mesh terms] OR "Cattle Diseases"[MeSH terms] OR "cattle"[tiab] AND Americas[MeSH Terms:noexp] OR Latin America[Mesh] OR Latin America*[tiab] OR Latin America*[ad] OR Latinoamerica*[ad] OR Latinoamerica*[tiab]

PUBMED OR Hispanoamerica*[ad] OR Hispanoamerica*[tiab] OR Iberoamerica*[ad] OR Iberoamerica*[tiab] OR Ibero Americ*[ad] OR Ibero Americ*[tiab] OR Panamerican*[ad] OR Panamerican*[tiab] OR Central America[Mesh] OR Central America*[ad] OR Central America*[tiab] OR Centroamerica*[ad] OR Centroamerica*[tiab] OR South America[Mesh] OR South America*[ad] OR South America*[tiab] OR Southamerica*[tiab] OR Sudamerica*[ad] OR Sudamerica*[tiab] OR America del sur[ad] OR Caribbean Region[Mesh] OR Caribbean[ad] OR Caribbe*[tiab] OR Caribe*[ad] OR Caribe*[tiab] Argentina[Mesh] OR Argentin*[ad] OR Argentin*[tiab] OR Argentina[pl] OR Bolivia[Mesh] OR Bolivia*[ad] OR Bolivia*[tiab] OR Bolivia[pl] OR Brazil[Mesh] OR Brazil*[ad] OR Brazil[tiab] OR Brasil*[ad] OR Brazil[pl] OR Colombia[Mesh] OR Colombia*[ad] OR Colombia*[tiab] OR Colombia[pl] OR Chile[Mesh] OR Chile*[ad] OR Chile*[tiab] OR Chile[pl] OR Ecuador[Mesh] OR Ecuador*[ad] OR Ecuador*[tiab] OR Ecuador[pl] OR Paraguay[Mesh] OR Paraguay*[ad] OR Paraguay*[tiab] OR Paraguay[pl] OR Peru[Mesh] OR Peru*[ad] OR Peru*[tiab] OR Peru[pl] OR Uruguay[Mesh] OR Uruguay*[ad] OR Uruguay*[tiab] OR Uruguay[pl] OR Venezuela[Mesh] OR Venezuel*[ad] OR Venezuel*[tiab] OR Venezuela[pl] OR Costa Rica[Mesh] OR Costa Ric*[ad] OR Costa Ric*[tiab] OR Costarric*[ad] OR Costarric*[tiab] OR Costaric*[tiab] OR Costa Rica[pl] OR El Salvador[Mesh] OR Salvador*[ad] OR Salvador*[tiab] OR El Salvador[pl] OR Guatemala[Mesh] OR Guatemal*[ad] OR Guatemal*[tiab] OR Guatemala[pl] OR Honduras[Mesh] OR Hondur*[ad] OR Hondur*[tiab] OR Honduras[pl] OR Nicaragua[Mesh] OR Nicaragu*[ad] OR Nicaragua[pl] OR Nicaragu*[tiab] OR Panama[Mesh] OR Panam*[ad] OR Panam ${ }^{*}\left[\right.$ tiab] OR Panama[pl] OR Mexico[Mesh] OR Mexic*[ad] OR Mexic ${ }^{*}[t i a b]$ OR Mejic*[ad] OR Mexico[pl] OR Cuba[Mesh] OR Cuba*[ad] OR Cuba*[tiab] OR Cuba[pl] OR Dominican Republic[Mesh] OR Dominic*[ad] OR Dominic*[tiab] OR Dominican Republic[pl] OR Haiti[Mesh] OR Haiti*[ad] OR Haiti*[tiab] OR Haiti[pl] OR Jamaica[Mesh] OR Jamaic*[ad] OR Jamaic*[tiab] OR Jamaica[pl] AND (“2003/01/01”[PDAT] : "3000/12/31"[PDAT])

(TW: "Salmonella" OR "Salmonella Infections" OR "Infecções por Salmonella" OR "Infecciones por Salmonella" OR "salmonelosis" OR "salmonellosis" OR "Salmonella Food Poisoning" OR "Intoxicación Alimentaria por Salmonella" OR "Intoxicação Alimentar por Salmonella" OR "Salmonella entérica" OR "Salmonella enteritidis" OR "Salmonella typhimurium" OR "S. enteritidis" OR "S. typhimurium") AND (TW: "microbial sensitivity tests" OR "Pruebas de Sensibilidad Microbiana" OR "Testes de Sensibilidade Microbiana" OR "Drug Resistance, Bacterial" OR "Farmacorresistencia Bacteriana" OR "Farmacorresistência Bacteriana" OR "Drug Resistance, Microbial" OR OR "Farmacorresistencia Microbiana" OR "Resistência Microbiana a Medicamentos" OR (("anti-infective agents" OR ("anti-infective" AND "agents") OR "antimicrobial" OR "antibiotic" OR "Bacterial" OR "microbial") AND ("resistance" OR "drug resistance, multiple" OR ("sensitivity" AND "tests")))) AND (TW: "animals" OR "animals " OR "animais" OR "animal" OR "abattoirs" OR "abattoir" OR "mataderos" OR "matadouros" OR "slaughterhouse" OR "slaughterhouses"

LILACS OR "slaughter" OR "butcher shop" OR "food microbiology" OR ("food" AND "microbiology") OR "microbiología de alimentos" OR "Pork" OR "sausage" OR "poultry" OR "Aves de Corral" OR "Aves Domésticas" OR "Poultry Diseases" OR "Enfermedades de las Aves de Corral" OR "Doenças das Aves Domésticas" OR "Productos Avícolas" OR "Produtos Avícolas" OR "Aviculture" OR "Avicultura" OR "pig" OR "chicken" OR "sausages" OR "poultries" OR "pigs" OR "chickens" OR "hens" OR "hen" OR "Pollos" OR "Pollo" OR "Galinhas" OR "eggs" OR "egg" OR "Huevo" OR "Huevos" OR "Ovos" OR "cerdos" OR "cerdo" OR "Porcinos" OR "suínos" OR "swine" OR "food pathogen" OR ("food" AND "pathogen") OR "patógeno alimentar" OR "patogeno de alimentos" OR "food surveillance" OR ("food" AND "surveillance") OR "vigilância alimentar" OR "vigilancia de lo alimentos" OR "meat" OR "Carne" OR "Productos de la Carne" OR "Produtos da Carne" OR "Swine Diseases" OR ("Swine" AND "Diseases") OR "Chickens/microbiology" OR "Eggs/microbiology" OR "Meat/microbiology" OR "Sheep, Domestic/microbiology" OR "Cattle/microbiology" OR "Swine/microbiology" OR "Poultry/microbiology" OR "Cattle Diseases" OR "cattle") 
Anexo 2. Herramienta de calidad adaptada para la evaluación de estudios seleccionados en la presente revisión.

\begin{tabular}{|c|c|c|c|c|c|c|}
\hline Ítems & Preguntas a evaluar & MB & B & $\mathbf{R}$ & $\mathbf{M}$ & NI \\
\hline \multirow{2}{*}{ 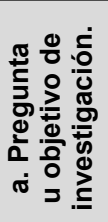 } & $\begin{array}{l}\text { 1. ¿En la formulación de la pregunta o del objetivo se menciona } \\
\text { adecuadamente el alimento y bacteria de estudio? }\end{array}$ & & & & & \\
\hline & $\begin{array}{l}\text { En resumen, el estudio se basa en una pregunta de investigación } \\
\text { claramente definida. }\end{array}$ & & & & & \\
\hline \multirow{3}{*}{ 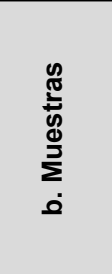 } & $\begin{array}{l}\text { 2. ¿Se indican los criterios de inclusión y de exclusión de las } \\
\text { muestras estudiados así como las fuentes y los métodos de } \\
\text { selección? }\end{array}$ & & & & & \\
\hline & $\begin{array}{l}\text { 3. ¿Los criterios de selección son adecuados para dar } \\
\text { respuesta a la pregunta o el objetivo del estudio? }\end{array}$ & & & & & \\
\hline & $\begin{array}{l}\text { En resumen, la muestra es adecuada, minimizándose la } \\
\text { probabilidad de sesgo de selección. }\end{array}$ & & & & & \\
\hline \multirow{5}{*}{ 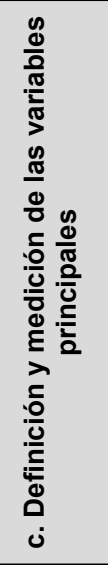 } & $\begin{array}{l}\text { 4. ¿Se exponen claramente cuáles son las variables de } \\
\text { resultados? }\end{array}$ & & & & & \\
\hline & $\begin{array}{l}\text { 5. ¿Las variables principales tienen una adecuada definición } \\
\text { conceptual (teórica) y operacional (escala de medición, sistema } \\
\text { de clasificación, etc.)? }\end{array}$ & & & & & \\
\hline & $\begin{array}{l}\text { 6. ¿Los instrumentos de medición de las variables principales } \\
\text { tienen validez y fiabilidad conocidas y adecuadas (se citan las } \\
\text { pruebas y métodos utilizados en los estudios que lo analizaron)? }\end{array}$ & & & & & \\
\hline & $\begin{array}{l}\text { 7. ¿Las técnicas de medición y recolección de las variables } \\
\text { principales se describen suficientemente, son adecuadas } \\
\text { (incluyendo aislamientos, serotipificación y pruebas de } \\
\text { sensibilidad antibiótica)? }\end{array}$ & & & & & \\
\hline & $\begin{array}{l}\text { En resumen, la medición y la recolección de las variables } \\
\text { principales se realizó de forma adecuada; minimizándose la } \\
\text { probabilidad de sesgos de información. }\end{array}$ & & & & & \\
\hline \multirow{4}{*}{ 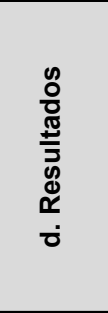 } & $\begin{array}{l}\text { 8. ¿Se incluyen resultados de todas las observaciones y se } \\
\text { indica el número de datos no disponibles? }\end{array}$ & & & & & \\
\hline & $\begin{array}{l}\text { 9. ¿Se presentan los resultados planteados en los objetivos y } \\
\text { todos los de interés, de manera clara y comprensible? }\end{array}$ & & & & & \\
\hline & $\begin{array}{l}\text { 10. ¿Se presentan estimaciones de la precisión de los } \\
\text { resultados (p. ej. intervalos de confianza)? }\end{array}$ & & & & & \\
\hline & $\begin{array}{l}\text { En resumen, los resultados están bien descritos, son útiles y } \\
\text { precisos. }\end{array}$ & & & & & \\
\hline \multirow{4}{*}{ 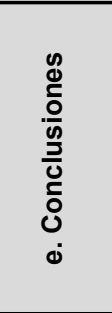 } & $\begin{array}{l}\text { 11. ¿Las conclusiones dan respuesta a los objetivos del } \\
\text { estudio? }\end{array}$ & & & & & \\
\hline & $\begin{array}{l}\text { 12. ¿Las conclusiones presentadas se basan en los resultados } \\
\text { obtenidos? }\end{array}$ & & & & & \\
\hline & $\begin{array}{l}\text { 13. ¿Los resultados del estudio son aplicables a la población } \\
\text { en interés? }\end{array}$ & & & & & \\
\hline & $\begin{array}{l}\text { En resumen, los resultados del estudio son generalizables a la } \\
\text { población y contexto en que interesa aplicarlos. }\end{array}$ & & & & & \\
\hline \multirow{4}{*}{ 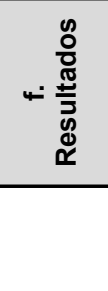 } & $\begin{array}{l}\text { 14. ¿Se menciona la fuente de financiación del estudio o los } \\
\text { autores declaran la existencia o ausencia de conflictos de } \\
\text { intereses? }\end{array}$ & & & & & \\
\hline & $\begin{array}{l}\text { En resumen, los conflictos de intereses no condicionan los } \\
\text { resultados ni las conclusiones del estudio. }\end{array}$ & & & & & \\
\hline & VALORACIÓN SUMARIA & ALTA & & MEDIA & & BAJA \\
\hline & Global de la calidad del estudio. & & & & & \\
\hline
\end{tabular}

